\title{
Heterogenous nuclear ribonucleoprotein Q increases protein expression from HIV-1 Rev-dependent transcripts
}

\author{
Michelle Vincendeau ${ }^{1 \dagger}$, Daniel Nagel ${ }^{1 \dagger}$, Jara K Brenke ${ }^{1,2}$, Ruth Brack-Werner ${ }^{1}$ and Kamyar Hadian ${ }^{1,2^{*}}$
}

\begin{abstract}
Background: Heterogenous nuclear ribonucleoproteins (hnRNPs) control many processes of the gene expression machinery including mRNA transcription, splicing, export, stability and translation. Recent data show interaction of the HIV-1 Rev regulatory protein with a subset of hnRNP proteins, that includes hnRNP Q, suggesting that hnRNPs can contribute to regulation of HIV-1 gene expression by Rev.

Findings: In this work we address the effect of hnRNP Q on Rev-dependent gene expression. We show that hnRNP Q overexpression increased levels of proteins produced from a Rev-dependent reporter gene in the presence of Rev. Increased protein levels did not correlate with changes in either the levels or the nucleocytoplasmic distribution of Rev-dependent reporter mRNAs. Similar observations were made in persistently HIV-1 infected HeLa cells. In these cells, hnRNP Q overexpression increased levels of the HIV-1 Gag-p24 protein, while levels of viral Rev-dependent mRNAs were not affected.

Conclusion: Our data indicate that hnRNP Q can stimulate the protein production of Rev-dependent mRNAs without changing mRNA levels and mRNA export, respectively. This suggests that hnRNP Q can boost HIV gene expression at the level of protein production.
\end{abstract}

Keywords: Rev, hnRNP Q, SYNCRIP, HIV-1 replication, Posttranscriptional regulation

\section{Findings}

HIV-1 Rev is critical for HIV-1 replication. Rev recognizes the Rev response element (RRE) in partly and unspliced viral RNAs. Binding of Rev to the RRE counteracts negative effects of instability elements (INS) in viral RNA molecules and promotes their transport to the cytoplasm [1-3]. Additionally, the Rev protein is involved in many other processes, e.g. RNA splicing [4], translation of viral RNAs [5,6] and packaging of viral particles [7]. Rev comprises different functional domains responsible for RNA binding/nuclear localization (nuclear localization signal, NLS) [8], nuclear export/transactivation (nuclear export

\footnotetext{
* Correspondence: Kamyar.hadian@helmholtz-muenchen.de ${ }^{\dagger}$ Equal contributors

'Institute of Virology, Helmholtz Zentrum München, Research Center for Environmental Health, Ingolstaedter Landstraße 1, 85764, Neuherberg, Germany

${ }^{2}$ Institute of Molecular Toxicology and Pharmacology/Assay Development and Screening Platform - Helmholtz Zentrum München, Research Center for Environmental Health, Ingolstaedter Landstraße 1, 85764, Neuherberg, Germany
}

\section{Biomed Central

(c) 2013 Vincendeau et al.; licensee BioMed Central Ltd. This is an Open Access article distributed under the terms of the Creative Commons Attribution License (http://creativecommons.org/licenses/by/2.0), which permits unrestricted use, distribution, and reproduction in any medium, provided the original work is properly cited. signal, NES) [9] and multimerization of the Rev-protein (oligomerization signals) [2]. Rev interacts with many cellular partners, including Exportin 1 (CRM1) and host cell RNA binding factors like DEAD/H box proteins [10]. In addition, we recently showed that the N-terminus of Rev is able to interact with several hnRNPs [11], a set of RNA binding proteins involved in multiple cellular processes [12]. These interactions seem to play an important role in connecting the diverse functions of Rev during viral gene expression with cellular processes.

The human hnRNP Q protein (synonym: SYNCRIP, Gry-rbp, NSAP1) exists in three isoforms (Q1, Q2 and Q3), which are generated from the same gene by alternative splicing [13]. The full-length protein (Q3) consists of 623 amino acids and contains three RNA-binding domains (RBD), two NLS and a C-terminal Arginine-rich motif (RGG-domain) [13]. HnRNP Q is important for efficient pre-mRNA splicing [13] and can impact mRNA stability $[14,15]$. Furthermore, hnRNP Q is capable of influencing IRES-dependent mRNA translation [16-18]. 
In the HIV system, hnRNP Q was recently shown to be part of a group of cellular proteins that binds to a segment of HIV-1 RNA containing splice acceptor site A7 [19] located near the RRE. Interestingly, the group of proteins binding to this region also included translational factors. In a recent study we could demonstrate that hnRNP Q interacts with HIV-1 Rev and is able to positively affect HIV replication, with knockdown of hnRNP Q decreasing viral production [11]. In this Short Report we now present additional research data that strengthen the previously published positive effects of hnRNP Q on HIV-1 replication.

We first used a Rev-dependent reporter assay $[3,20]$ to investigate the influence of hnRNP Q on Rev-dependent reporter protein production. Assays were performed in
HeLaTatROD cells that contain a stably integrated Revreporter gene encoding the DsRed protein and constitutively produce Tat for LTR-dependent transcription of the reporter gene [3]. HeLaTatROD cells were co-transfected with expression plasmids containing the full-length hnRNP Q cDNA sequence fused to sequences encoding a cyan fluorescent tag (pC-hnRNP Q-CYN) and with plasmids for expression of HIV-1 RevGFP (pCsRevsg143; [21]). Expression was verified in HeLa cells (Figure 1A), with hnRNP Q-CYN showing the steady-state nuclear localization pattern described for hnRNP Q [13]. Control transfections were performed in parallel with plasmids (pC-CYN; [11]) expressing only the cyan fluorescent tag. Transfections were performed with the FugeneHD transfection reagent (Roche). Interaction of hnRNP Q with Rev

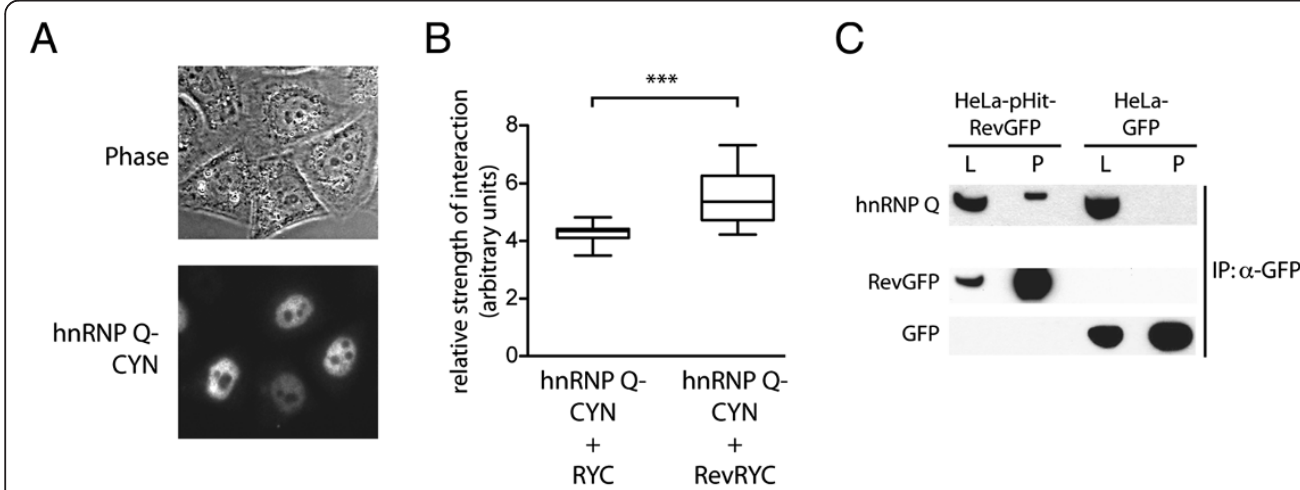

D

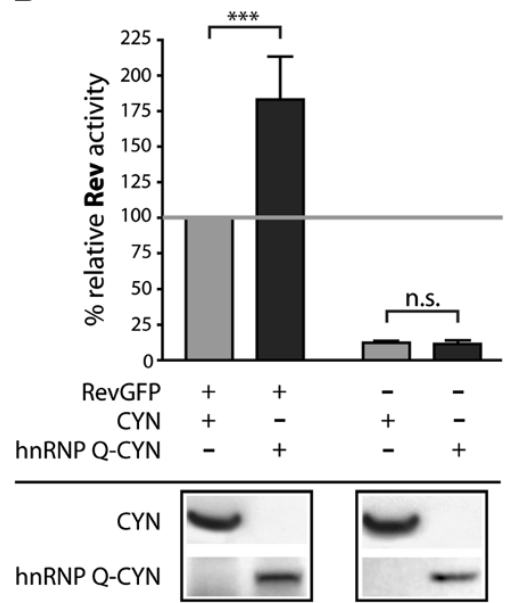

E

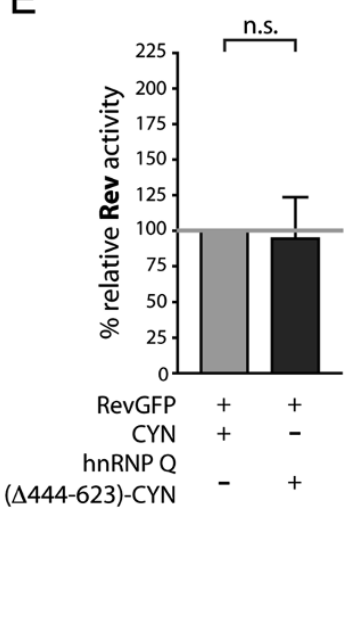

$\mathrm{F}$

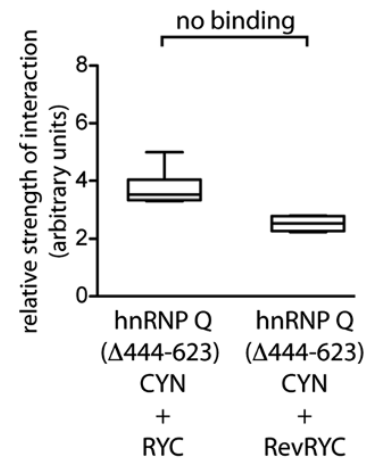

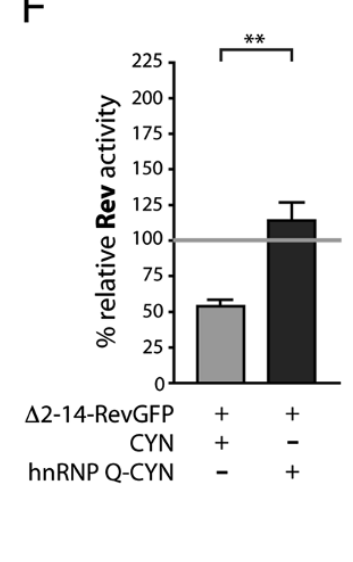

Figure 1 HnRNP Q induces Rev-dependent reporter protein production. A) Expression of hnRNP Q-CYN in HeLa cells. B) Rev-mRFP-YFP-C (Rev-RYC) and hnRNP Q-CFP-YFP-N (hnRNP Q-CYN) significantly interact in exBIFC assays in HeLa cells. C) Immunoprecipitation of Rev-GFP with an anti-GFP antibody specifically co-precipitates endogenous hnRNP Q. D) HeLaTatROD cells stably expressing Tat and a Rev-dependent reporter construct were used to evaluate the effect of hnRNP Q on Rev-dependent protein expression. Co-expression of Rev and hnRNP Q-CYN increased reporter protein production by approx. 2-fold compared to the control (CYN). Importantly, this effect was not detectable in the absence of Rev. Expression of hnRNP Q-CYN and CYN proteins were detected by Western Blotting using an anti-GFP antibody. E) An hnRNP Q deletion mutant lacking aa 444-623 (hnRNP Q ( $\triangle 444-623)-C Y N)$ was not able to enhance Rev-dependent reporter protein production (left graph). Moreover, this mutant lacks the capacity to bind Rev in exBIFC assays (right graph). (F) A Rev mutant lacking the amino acids 2-14 ( $\triangle 2-14 R e v G F P)$ showed a $50 \%$ decreased activity when compared to Rev-GFP. This activity was enhanced by ectopic expression of hnRNP Q. Bars represent the means of at least 4 independent experiments and standard deviations are indicated. $p$-values: ${ }^{* *}=p \leq 0.001 ;$ n.s. $=$ not significant $(p>0.05)$. 
was demonstrated by two assays, i.e. exBIFC (extended bimolecular fluorescence complementation) [22] and coimmunoprecipitation (co-IP) (Figure $1 \mathrm{~B}$ and $\mathrm{C}$ ). For exBIFC, Rev was expressed as a fusion protein with the $\mathrm{N}$-terminal half of YFP, while hnRNP Q was linked to the C-terminal half of YFP. The interaction of Rev and hnRNP Q results in complementation of a fluorescing YFP molecule. Co-IP studies were performed with an antibody directed against GFP, thereby precipitating RevGFP containing complexes.

As expected, expression of Rev substantially increased reporter protein production (Figure 1D). Co-expression of RevGFP with hnRNP Q-CYN significantly boosted reporter protein levels even further (approx. 2-fold) compared to the CYN-control (Figure 1D). Similar results were obtained with a plasmid expressing hnRNP Q without the CYN-tag (data not shown), confirming the specific influence of hnRNP $Q$ on reporter protein production. In comparison, hnRNP A1 was not as potent as hnRNP $\mathrm{Q}$ to induce reporter protein production (Additional file 1A). Interestingly, hnRNP Q did not increase reporter protein production in the absence of Rev (Figure 1D). In addition, a hnRNP Q deletion mutant lacking the RGG domain from amino acids 444-623 (hnRNP Q-( $(444-623)-C Y N)$ was not able to boost reporter expression and did not bind Rev in exBIFC assays (Figure 1E). As a control, we explored the effect of hnRNP Q on a GagCTE reporter [23]. This reporter drives Gag production under the control of a LTR promoter and its expression is independent of Rev, but rather uses the TAP/CTE pathway. We could detect a small increase in Gag production through the GagCTE reporter, but notably less pronounced when compared to our Rev-dependent reporter construct (compare Figure 1D and Additional file 2). Further, a Rev mutant lacking the $\mathrm{N}$-terminal 14 amino acids $(\Delta 2-14-\mathrm{Rev})$, a region involved in the binding to different hnRNPs [11], showed reduced ability to induce Rev-dependent reporter protein production. Surprisingly, hnRNP Q was able to further increase levels of reporter proteins driven by the $\Delta 2-14-\operatorname{Rev}$ mutant indicating that hnRNP Q may also affect reporter protein production by mechanisms independent of Rev-hnRNP Q interaction (Figure 1F). Taken together, our data demonstrate that hnRNP Q is capable of enhancing Rev-mediated reporter protein production.

To further analyze reporter activation by hnRNP Q, we evaluated the effects of hnRNP Q on mRNA levels. Again HeLaTatROD cells were co-transfected with a RevGFP expression plasmid together with CYN and hnRNP Q-CYN expression plasmids, respectively. Total RNAs were extracted, reverse transcribed into cDNA (Invitrogen Superscript cDNA reverse transcription kit) and analyzed by quantitative real-time PCR as previously described [21] using specific primers for amplification of the reporter RNAs (forward primer 5'-CGAGCTCGG TACCCCAAGGCAAAGAGAAGAGTGG-3'; reverse primer 5' -CAATAGCCCTCAGCAAATTGTTCTGCTGC$\left.3^{\prime}\right)$ (Figure 2A). For data analysis, the $C p$ values were normalized to the levels of human RNA Polymerase II. Our results show that reporter mRNA levels are not significantly affected by hnRNP Q expression when compared to the CYN control. This observation was independent of the expression of Rev (Figure 2B). Next, we analyzed the impact of hnRNP Q on nuclear export of reporter mRNAs. To this end, extracts of transfected cells (expressing RevGFP together with hnRNP Q-CYN or with the CYN control) were separated into nuclear and cytoplasmic fractions using the PARIS Kit (Ambion). RNAs from both fractions were individually purified, reverse transcribed and reporter transcripts were analyzed by quantitative RT-PCR as described above. Purity of nuclear and cytoplasmic fractions was verified by Western Blot analysis using histone $\mathrm{H} 2 \mathrm{~A}$ as a nuclear and GAPDH as a cytoplasmic marker. Both fractions showed fairly no cross-contamination (Figure 2C). Investigation of nuclear and cytoplasmic reporter mRNAs by qRT-PCR revealed no increase of reporter transcripts in the cytoplasmic compartment after hnRNP Q overexpression compared to the CYN control (Figure 2C), suggesting that hnRNPQ overexpression does not influence nuclear export of reporter mRNAs. In contrast, hnRNP A1 clearly increased levels of nuclear reporter mRNA (Additional file 1B).

To study the effects of hnRNP Q in the context of complete HIV-1 replication, we analyzed the influence of hnRNP Q overexpression on levels of p24Gag produced by persistently HIV-1 infected HeLa cells (LC5-HIV) [24]. p24 protein levels were quantified by ELISA as previously described $[11,21]$. In line with our data showing increased Rev-dependent reporter protein production by hnRNP Q (Figure 1D), ectopic hnRNP Q expression significantly increased intra- and extracellular p24 protein levels by LC5-HIV cells (Figure 3A). Hence, hnRNP Q is also able to enhance production of late viral gene products in a persistently infected cell system. A slight increase in p24 protein production was also detected after ectopic expression of hnRNP A1 (Additional file 1C). To evaluate whether hnRNP $\mathrm{Q}$ alters the levels of Rev-dependent HIV-1 transcript classes, we quantified levels of different HIV-1 transcript species in LC5-HIV cells expressing hnRNP Q-CYN. To this end we performed qRT-PCR with primers (Figure $3 \mathrm{~B}$ ) that specifically amplify either Revdependent late transcripts (i.e. unspliced and singly spliced RNAs: forward primer 5'-GCCCCTCCCATCAGTG GAC-3' and reverse primer 5' - GCCTTGGTGGGTCG TACTCCTAATGG-3') or Rev-independent early transcripts (i.e. completely spliced RNAs: forward primer $5^{\prime}$ - 
A

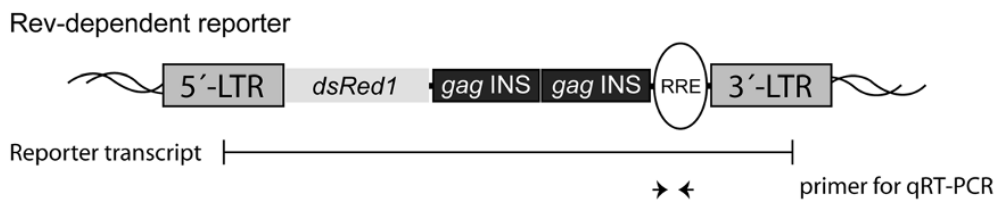

B

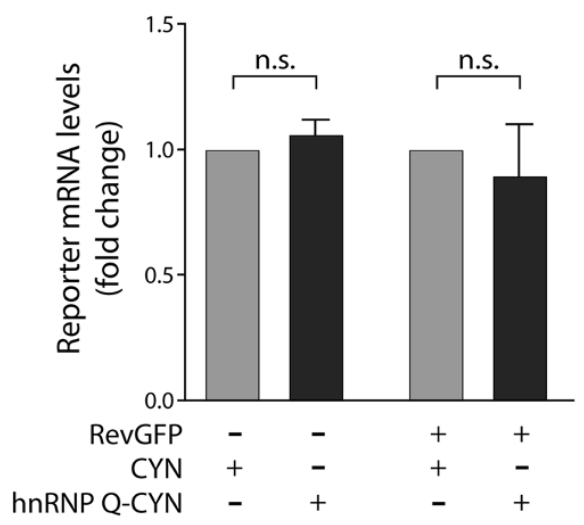

C
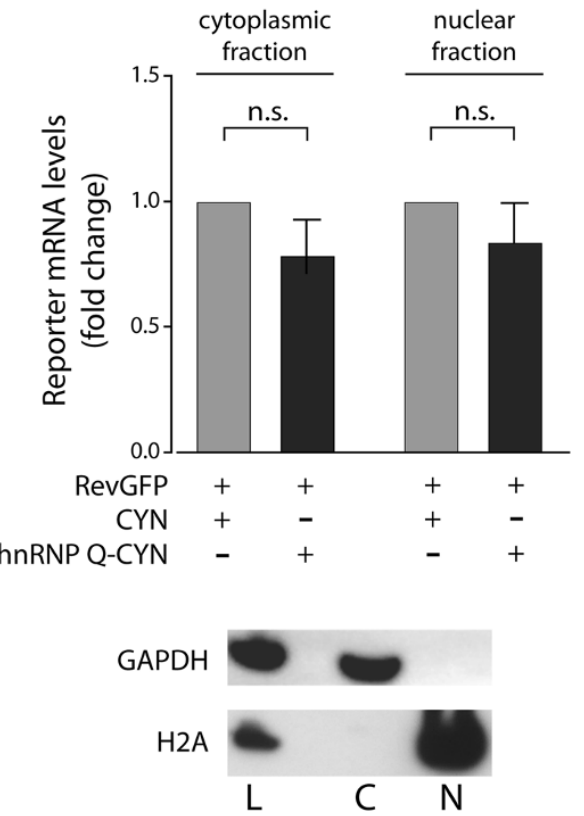

Figure 2 Overexpression of hnRNP Q does not affect the levels and the nucleocytoplasmic distribution of Rev-dependent reporter mRNAs. A) Schematic organization of the Rev-dependent reporter construct. The 5'-LTR directs transcription of the DsRed reporter gene. The reporter mRNA contains Rev-dependent regulatory elements (i.e. gag-derived instability elements and a Rev-response element (RRE)). Primers for qRT-PCR analyses bind within the RRE region. B) Total reporter RNA levels were investigated after expression of hnRNP Q-CYN or the CYN control in the presence or absence of Rev. Overexpression of hnRNP Q did not change total levels of reporter mRNA independent of Rev expression. C) Reporter RNA levels were also analyzed after separation of cellular lysates into cytoplasmic and nuclear fractions. Overexpression of hnRNP Q did not enhance export of reporter mRNAs. H2A and GAPDH were used to evaluate purity of cytoplasmic and nuclear fractions, respectively. Bars represent the means of at least 3 independent experiments and standard deviations are indicated. $p$-values: n.s. $=$ not significant $(p>0.05)$.

CTCTATCAAAGCAACCCACCTCCCAA-3' and reverse primer 5'-GCGGTGGTAGCTGAAGAGGCACAGG-3') or all HIV-1 transcripts (forward primer 5'-CCAGTCAC ACCTCAGGTACCTTTAAGACC-3' and reverse primer 5'-GTGTGTGGTAGATCCACAGATCAAGG-3'). HIV-1 transcript levels were normalized to human RNA Polymerase II mRNA levels. The results of these qRT-PCR analyses demonstrate that hnRNP $\mathrm{Q}$ does not significantly alter levels of Rev-dependent or Rev-independent transcript classes (Figure 3C) or of total HIV-1 transcripts (Figure 3D). These results indicate that hnRNP Q overexpression does not affect quantities of Rev-dependent mRNAs in HIVproducing cells, and agree with the results of the analysis of reporter transcripts (Figure 2B).

In this Short Report we addressed the effect of hnRNP Q on Rev-dependent reporter production and HIV-1 replication. For overexpression we used the full-length hnRNP Q coding sequence. Overexpression of hnRNP Q increased protein levels produced from Rev-dependent transcripts without affecting amounts or nucleocytoplasmic distribution of Rev-dependent transcripts. This point is remarkable as hnRNP Q is known to influence Hepatitis $C$ virus RNA replication [25], splicing events [13] and mRNA stability $[14,15]$. The increase in p24 production is congruent with our recent findings that knockdown of hnRNP $\mathrm{Q}$ in persistently infected astrocytes leads to diminished p24 production [11]. Of note, the influences by hnRNP Q on the Rev-dependent reporter protein production were visibly higher when compared to a GagCTE reporter indicating a specific effect of hnRNP Q on the Rev/RRE axis. Moreover, hnRNP Q had a bigger effect on Rev-dependent reporter protein production than hnRNP A1 and the effects by these two hnRNPs were distinct of each other. While hnRNP Q did not affect mRNA levels, hnRNP A1 markedly increased reporter 

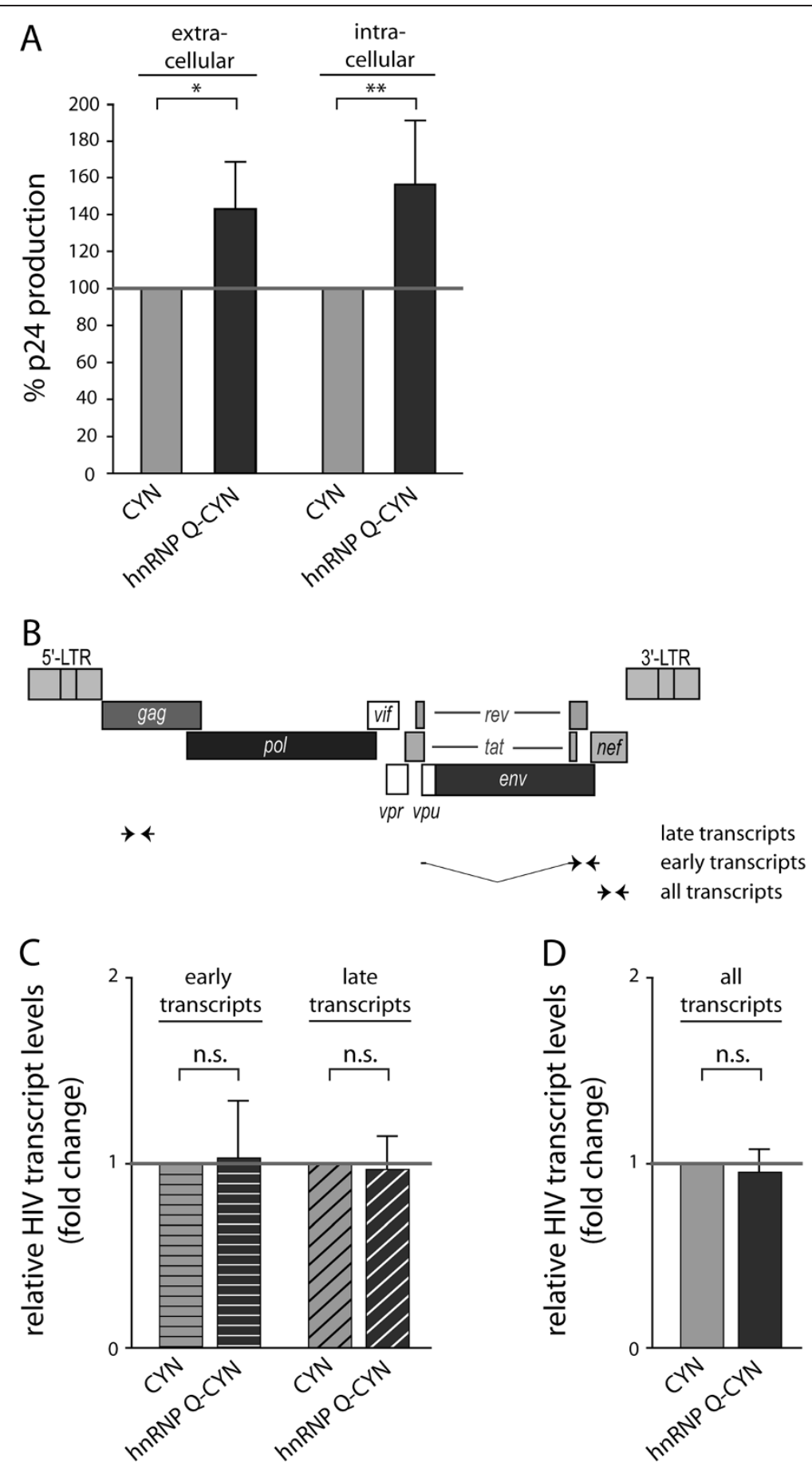

Figure 3 HnRNP Q overexpression elevates Gag-p24 production without affecting levels of HIV-1 transcripts. A) Chronically HIV-1 infected HeLa cells (LC5-HIV) were used to evaluate the effects of hnRNP Q overexpression on HIV-1 replication. Using ELISA, p24 protein levels were analyzed in LC5-HIV cells ectopically expressing hnRNP Q-CYN or CYN. The results revealed that both extra- and intracellular p24 protein levels are elevated by hnRNP Q overexpression. B) Specific primers (indicated in the scheme of the HIV-1 genome) were designed to distinguish between Rev-dependent late transcripts (i.e. unspliced and singly-spliced), and Rev-independent early transcripts (i.e. completely spliced) and all transcripts. The primers for detection of late transcripts were placed into the gag ORF and for detection of all transcripts into the nef ORF. We used a 5' spanning primer bridging the two rev exons and a 3' primer located at the end of the second rev exon to quantify early transcripts by qRT-PCR. C) Quantitative analyses of HIV transcripts showed that levels of neither early nor late transcripts are affected by ectopic expression of hnRNP Q in chronically HIV-1 infected cells. D) Analysis of total levels of HIV-1 transcripts confirmed that overexpression of hnRNP Q did not affect HIV-1 transcript levels. p-values: ${ }^{*}=p \leq 0.05 ;{ }^{* *}=p \leq 0.01 ;$ n.s. $=$ not significant $(p>0.05)$. 
mRNA levels in the nucleus. One possibility to explain these hnRNP A1 effects is that hnRNP A1 is able to stabilize INS containing mRNAs [26]. Over time the elevated number of reporter mRNAs lead to an increased protein production. However, hnRNP A1 has also other influences on viral replication (e.g. on the level of splicing) that could lead to the induced reporter protein and p24 production.

Interestingly, the influence of hnRNP Q on protein production from a Rev dependent reporter gene was only visible in the presence of HIV-1 Rev. Rev interaction with hnRNP Q was shown to involve the N-terminus of Rev [11]. Surprisingly, we still see a positive effect of hnRNP Q on the activity of a Rev-deletion mutant lacking the $\mathrm{N}$-terminus. This could be due to secondary binding sites in Rev for hnRNP Q since we previously reported that deletion of the N-terminus of Rev significantly diminished, but did not abrogate binding of Rev to hnRNP Q [11]. However, we cannot exclude that there is also an activity of hnRNP Q on HIV-1 protein production independent of direct binding to Rev. In future studies we will decipher the mechanisms behind the mutual as well as individual impacts of Rev and hnRNP proteins to regulate HIV-1 replication.

Recently the Rev co-factors Sam68, eIF5A, hRIP and DDX3, which are essential for nuclear export of Revdependent mRNAs [7], were shown to be able to regulate HIV-1 replication on the translational level [27]. This expands the impact of multifunctional Revinteraction partners to processes in the cytoplasm. Our new data suggest that hnRNP Q could also be involved in the translational control of HIV-1 replication. Indeed, hnRNP $Q$ was shown to influence translation of different mRNAs, including $\mathrm{HCV}$ mRNA [17], BiP mRNA [16] and Rev-erb alpha within circadian oscillation [18] proving its presence and function in the cytoplasmic compartment. The very diverse set of hnRNP proteins comprise a variety of cellular functions (mRNA transcription, splicing, trafficking, stability, translation, etc.) inside the nucleus as well as the cytoplasm [28,29] and many hnRNPs are known to regulate HIV-1 replication (discussed in [11] and $[30,31])$. Thus, hnRNP Q may also recruit other hnRNPs to co-activate HIV-1 replication. Future studies will address the role of hnRNP Q in the translation of HIV-1 proteins.

Taken together, we demonstrate supporting data that hnRNP Q increases p24 production in persistently infected cells and Rev-dependent reporter protein production. These effects are not associated with changes in RNA levels or nucleocytoplasmic distribution of Rev-dependent mRNAs, suggesting that hnRNP Q can contribute to positive effects on HIV-1 protein production.

\section{Additional files}

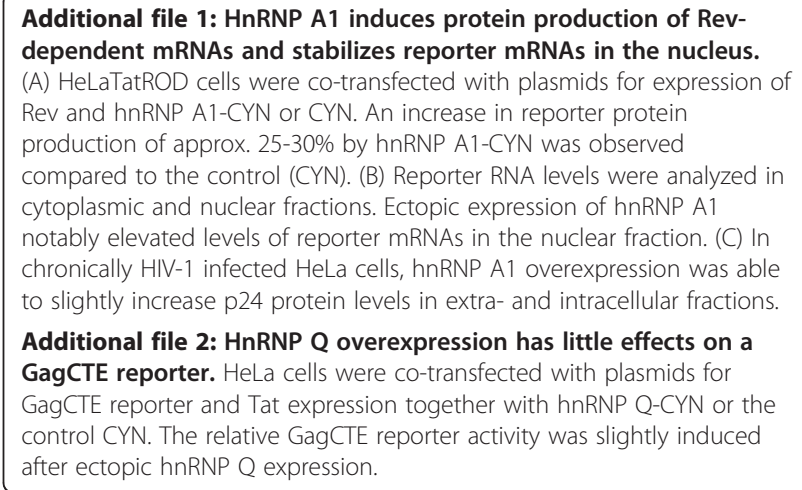

Competing interests

The authors declare that they have no competing interests.

Authors' contributions

$M V, D N, R B W$ and $K H$ designed the experiments. MV, DN, JB and $\mathrm{KH}$ performed the experiments. RBW and $\mathrm{KH}$ wrote the manuscript. All authors read and approved the final manuscript.

\section{Acknowledgements}

KH was supported by the German research council (DFG), grant 1710/1-3 to RBW. We thank Dr. Felber for providing us with plasmids for GagCTE reporter and Tat expression.

Received: 5 January 2012 Accepted: 19 March 2013 Published: 16 May 2013

\section{References}

1. Felber BK, Zolotukhin AS, Pavlakis GN: Posttranscriptional control of HIV-1 and other retroviruses and its practical applications. Adv Pharmacol 2007, 55:161-197.

2. Kjems J, Askjaer P: Rev protein and its cellular partners. Adv Pharmacol 2000, 48:251-298.

3. Wolff H, Brack-Werner R, Neumann M, Werner T, Schneider R: Integrated functional and bioinformatics approach for the identification and experimental verification of RNA signals: application to HIV-1 INS. Nucleic Acids Res 2003, 31:2839-2851.

4. Kammler S, Otte M, Hauber I, Kjems J, Hauber J, Schaal H: The strength of the HIV-1 3' splice sites affects Rev function. Retrovirology 2006, 3:89.

5. Arrigo SJ, Chen IS: Rev is necessary for translation but not cytoplasmic accumulation of HIV-1 vif, vpr, and env/vpu 2 RNAs. Genes Dev 1991, 5:808-819.

6. D'Agostino DM, Felber BK, Harrison JE, Pavlakis GN: The Rev protein of human immunodeficiency virus type 1 promotes polysomal association and translation of gag/pol and vpu/env mRNAs. Mol Cell Biol 1992, 12:1375-1386.

7. Groom HC, Anderson EC, Lever AM: Rev: beyond nuclear export. J Gen Virol 2009, 90:1303-1318.

8. Daugherty MD, Liu B, Frankel AD: Structural basis for cooperative RNA binding and export complex assembly by HIV Rev. Nat Struct Mol Biol 2010, 17:1337-1342.

9. Guttler T, Madl T, Neumann P, Deichsel D, Corsini L, Monecke T, Ficner R, Sattler M, Gorlich D: NES consensus redefined by structures of PKI-type and Rev-type nuclear export signals bound to CRM1. Nat Struct Mol Biol 2010, 17:1367-1376.

10. Naji S, Ambrus $G$, Cimermancic $P$, Reyes JR, Johnson JR, Filbrandt $R$, Huber MD, Vesely P, Krogan NJ, Yates JR 3rd, et al: Host cell interactome of HIV-1 Rev includes RNA helicases involved in multiple facets of virus production. Mol Cell Proteomics 2012, 11:M111 015313.

11. Hadian K, Vincendeau M, Mausbacher N, Nagel D, Hauck SM, Ueffing M, Loyter A, Werner T, Wolff H, Brack-Werner R: Identification of a heterogeneous nuclear ribonucleoprotein-recognition region in the HIV Rev protein. J Biol Chem 2009, 284:33384-33391. 
12. Dreyfuss G, Kim VN, Kataoka N: Messenger-RNA-binding proteins and the messages they carry. Nat Rev Mol Cell Biol 2002, 3:195-205.

13. Mourelatos Z, Abel L, Yong J, Kataoka N, Dreyfuss G: SMN interacts with a novel family of hnRNP and spliceosomal proteins. EMBO J 2001, 20:5443-5452.

14. Grosset C, Chen CY, Xu N, Sonenberg N, Jacquemin-Sablon H, Shyu AB: A mechanism for translationally coupled mRNA turnover: interaction between the poly $(\mathrm{A})$ tail and a c-fos RNA coding determinant via a protein complex. Cell 2000, 103:29-40.

15. Kim TD, Kim JS, Kim JH, Myung J, Chae HD, Woo KC, Jang SK, Koh DS, Kim $K T$ : Rhythmic serotonin $\mathrm{N}$-acetyltransferase mRNA degradation is essential for the maintenance of its circadian oscillation. Mo/ Cell Biol 2005, 25:3232-3246.

16. Cho S, Park SM, Kim TD, Kim JH, Kim KT, Jang SK: BiP internal ribosomal entry site activity is controlled by heat-induced interaction of NSAP1. Mol Cell Biol 2007, 27:368-383.

17. Kim JH, Paek KY, Ha SH, Cho S, Choi K, Kim CS, Ryu SH, Jang SK: A cellular RNA-binding protein enhances internal ribosomal entry site-dependent translation through an interaction downstream of the hepatitis $\mathrm{C}$ virus polyprotein initiation codon. Mol Cell Biol 2004, 24:7878-7890.

18. Kim DY, Woo KC, Lee KH, Kim TD, Kim KT: hnRNP Q and PTB modulate the circadian oscillation of mouse Rev-erb alpha via IRES-mediated translation. Nucleic Acids Res 2010, 38:7068-7078.

19. Marchand V, Santerre M, Aigueperse C, Fouillen L, Saliou JM, Van Dorsselaer A, Sanglier-Cianferani S, Branlant C, Motorin Y: Identification of protein partners of the human immunodeficiency virus 1 tat/rev exon 3 leads to the discovery of a new HIV-1 splicing regulator, protein hnRNP K. RNA Biol 2011, 8:325-342.

20. Wolff H, Hadian K, Ziegler M, Weierich C, Kramer-Hammerle S, Kleinschmidt A, Erfle V, Brack-Werner R: Analysis of the influence of subcellular localization of the HIV Rev protein on Rev-dependent gene expression by multi-fluorescence live-cell imaging. Exp Cell Res 2006, 312:443-456.

21. Vincendeau M, Kramer S, Hadian K, Rothenaigner I, Bell J, Hauck SM, Bickel C, Nagel D, Kremmer E, Werner T, et al: Control of HIV replication in astrocytes by a family of highly conserved host proteins with a common Rev-interacting domain (Risp). AIDS 2010, 24:2433-2442.

22. Wolff H, Hartl A, Eilken HM, Hadian K, Ziegler M, Brack-Werner R: Live-cell assay for simultaneous monitoring of expression and interaction of proteins. Biotechniques 2006, 41:688-690. 692.

23. Smulevitch S, Bear J, Alicea C, Rosati M, Jalah R, Zolotukhin AS, von Gegerfelt A, Michalowski D, Moroni C, Pavlakis GN, Felber BK: RTE and CTE mRNA export elements synergistically increase expression of unstable. Rev-dependent HIV and SIV mRNAs. Retrovirology 2006, 3:6.

24. Brack-Werner R, Kleinschmidt A, Ludvigsen A, Mellert W, Neumann M, Herrmann R, Khim MC, Burny A, Muller-Lantzsch N, Stavrou D, et al: Infection of human brain cells by HIV-1: restricted virus production in chronically infected human glial cell lines. AIDS 1992, 6:273-285.

25. Liu HM, Aizaki H, Choi KS, Machida K, Ou JJ, Lai MM: SYNCRIP (synaptotagmin-binding, cytoplasmic RNA-interacting protein) is a host factor involved in hepatitis C virus RNA replication. Virology 2009, 386:249-256.

26. Najera I, Krieg M, Karn J: Synergistic stimulation of HIV-1 rev-dependent export of unspliced mRNA to the cytoplasm by hnRNP A1. J Mol Biol 1999, 285:1951-1964.

27. Liu J, Henao-Mejia J, Liu H, Zhao Y, He JJ: Translational regulation of HIV-1 replication by HIV-1 Rev cellular cofactors Sam68, elF5A, hRIP, and DDX3. J Neuroimmune Pharmacol 2011, 6:308-321.

28. Han SP, Tang YH, Smith R: Functional diversity of the hnRNPs: past, present and perspectives. Biochem J 2010, 430:379-392.
29. Farina KL, Singer RH: The nuclear connection in RNA transport and localization. Trends Cell Biol 2002, 12:466-472.

30. Cochrane AW, McNally MT, Mouland AJ: The retrovirus RNA trafficking granule: from birth to maturity. Retrovirology 2006, 3:18.

31. Stoltzfus CM, Madsen JM: Role of viral splicing elements and cellular RNA binding proteins in regulation of HIV-1 alternative RNA splicing. Curr HIV Res 2006, 4:43-55.

doi:10.1186/1743-422X-10-151

Cite this article as: Vincendeau et al.: Heterogenous nuclear ribonucleoprotein Q increases protein expression from HIV-1 Rev-dependent transcripts. Virology Journal 2013 10:151.

\section{Submit your next manuscript to BioMed Central and take full advantage of:}

- Convenient online submission

- Thorough peer review

- No space constraints or color figure charges

- Immediate publication on acceptance

- Inclusion in PubMed, CAS, Scopus and Google Scholar

- Research which is freely available for redistribution

Submit your manuscript at www.biomedcentral.com/submit
C Biomed Central 